\title{
Evaluación del impacto de la educación superior en la iniciativa emprendedora
}

\author{
$\mathrm{M}^{\mathrm{a}}$ Pilar Flores ASENJo \\ Universidad Católica San Antonio de Murcia \\ pflores@ucam.edu \\ José PALAo Barberá \\ Universidad Católica San Antonio de Murcia \\ palao.barbera@telefonica.net
}

\section{Resumen}

Partiendo de los distintos enfoques del emprendimiento y las competencias que los definen, se pretende establecer un método para comprobar si el impacto de la educación en competencias genera el resultado esperado y si existe la posibilidad de integrar las acciones formativas y competencias en cualquier nivel de educación superior, independientemente del perfil de carrera del estudiante.

Palabras clave: Educación en emprendimiento, Teoría del Comportamiento Planeado, competencias, intención de emprender

\section{Assessing the impact of higher education on entrepreneurial intention}

\begin{abstract}
First it is analyzed entrepreneurial attitudes and the phases of the entrepreneurial process to propose a competence profile of entrepreneurial individual, and then, it is designed a method to analyze the impact of entrepreneurship education program on students' entrepreneurship skills and motivation.
\end{abstract}

Key Words: Entrepreneurship education, Planned Behavior Theory, education program, entrepreneurial intention

\section{Referencia normalizada:}

Flores Asenjo, M. P.; Palao Barberá, J. (2013) Evaluación del impacto de la educación superior en la iniciativa emprendedora. Historia y Comunicación Social. Vol. $18 \mathrm{~N}^{\mathrm{o}}$ Especial Noviembre. Págs. 377-386.

Sumario: 1. La iniciativa emprendedora y competencias. 1.1. Definición de las competencias según enfoques. 1.2. Competencias en emprendimiento e impacto en la educación. 2. Objetivo y metodología de la investigación. 3. Resultados. 4. Conclusiones. 5. Referencias Bibliográficas.

\section{La iniciativa emprendedora y competencias}

La iniciativa emprendedora, entendida como el descubrimiento de nuevas oportunidades en el mercado (Kirzner, 1979), creación de nuevas empresas (Gartner, 1985) o nuevos proyectos innovadores (Casson, 1982), es un elemento clave para el desa- 
rrollo económico y social, por su impulso de la innovación y la competitividad. Las principales causas del crecimiento del sistema económico son las acciones desarrolladas por los emprendedores de creación e innovación para responder a las oportunidades del entorno (Alda, 2012) introduciendo nuevas técnicas e innovaciones (Van Praag, 1999).

Según la Comisión Europea (2004), para lograr que la Unión Europea sea "la economía basada en el conocimiento más competitiva y dinámica del mundo", una de las competencias básicas es la iniciativa emprendedora. El desarrollo económico de los países depende en gran medida de su capacidad para crear nuevas empresas y puestos de trabajo (Holmgren y From, 2005; Parker, 2005; Wennekers et al, 2005; Van Praag, 2007; GEM, 2009).

Este marco ha llevado a que las iniciativas efectivas en relación a la formación en "creación de empresas" sean cada vez más relevantes en el diseño de los planes de acción políticos para el desarrollo económico (Comisión Europea, 2006 y 2008; OCDE, 2008), la creación de empleo (García et al, 2009) y los planes de estudios superiores de las Universidades.

Para el estudio de las competencias, aptitudes y habilidades asociadas al emprendimiento, los investigadores se han basado en los rasgos que diferencian a los emprendedores de los no emprendedores y en la forma en la que se toma la decisión de emprender.

\subsection{Definición de las competencias según enfoques}

En el enfoque de rasgos, a la hora de definir las características generalmente asociadas a las intenciones emprendedoras, se va a tener en cuenta tanto el emprendizaje independiente, entendido como la creación de una nueva empresa por un promotor independiente, como el corporativo, referido al fenómeno emprendedor dentro de una organización (intraemprendizaje, corporate entrepreneurship o corporate venturing).

La iniciativa, la creatividad, la independencia, la capacidad para aceptar retos o asumir riesgos moderados y responsabilidades, son rasgos que deben de caracterizar al emprendedor en cualquiera de los casos (Thomas y Mueller, 2000; Gürol y Atsan, 2006; Ulijn et al., 2007). Estas actitudes del emprendedor, que son elementos competenciales que mueven al sujeto a la acción y hacia el éxito en su desempeño (Yániz y Villadón, 2006), pueden parecer que ya están definidas en el individuo y que no existe posibilidad de ser aprendidas, pero sí es posible potenciar las habilidades propias del liderazgo, la capacidad de trabajo en equipo, la comunicación y las habilidades de negociación, así como las propias del ámbito del "saber hacer", como buscar información, planificar, organizar, tomar decisiones y buscar soluciones creativas (Cano et al, 2003) .

Según el enfoque del comportamiento, el emprendimiento debe ser visto como un proceso que se genera en el tiempo (Kyrö y Carrier, 2005), siendo la intención de emprender el primero de sus pasos (Lee y Wong, 2004). La teoría del comportamiento 
planificado establece tres factores motivacionales explicativos de la intención del comportamiento, que pueden ser aplicados al emprendimiento: las actitudes personales o grado en que los individuos evalúan positiva o negativamente ser emprendedor (Ajzen, 2002), y que incluye consideraciones afectivas como "me gusta..." y evaluativas como "me es más ventajoso/interesante..."; las normas subjetivas, que miden la presión social percibida hacia el comportamiento emprendedor, sobre todo de las personas de "referencia" del sujeto (familia, amigos, etc.); y el control del comportamiento percibido o percepción de la capacidad propia para ser emprendedor y poder controlar la acción de emprender.

Según un enfoque mixto, las actitudes son elementos competenciales que pueden ser un incentivo o una barrera (si no existen) para el emprendimiento (Yániz y Villardón, 2006), pero emprender implica desarrollar una acción, y su principal precursor es la intención y aquellos elementos relacionados con ella. Es necesario, por tanto, medir el impacto de la formación tanto en los rasgos que definen al emprendedor como en los tres factores relacionados con la intención de emprender.

El conocimiento sobre el emprendimiento o "creación de empresas" debería tener un efecto directo y significativo en la intención del emprendedor. La formación en empresas debe ir dirigida, según este enfoque, a modificar la intención emprendedora de los estudiantes, mejorar sus oportunidades de desarrollo profesional y laboral, estimular sus capacidades emprendedoras y prepararlos para un mercado laboral muy dinámico (Veciana, 1999).

\subsection{Competencias en emprendimiento e impacto de la educación}

Las necesidades creadas en el marco de la EEES y la necesidad de las economías y políticas actuales de crecimiento y creación de empleo hacen indispensable la integración de la educación en competencias de emprendimiento en todas las áreas de estudio, empleando técnicas participativas y creativas como la creación de microempresas, simulaciones empresariales, etc. En este sentido es cada vez más frecuente que en las Guías docentes de los Grados aparezca como objetivo la capacitación para el emprendimiento, y como competencias sistémicas la iniciativa y el espíritu emprendedor.

En la cultura organizativa actual existe la necesidad de reducir peso a los conocimientos técnicos en favor de las habilidades directivas que puedan mejorar la actuación de trabajadores y directivos "en las tres áreas básicas del quehacer del directivo del futuro: conocer, ser y hacer" (Wandosell y Palao, 2012: 195). Esta necesidad del mercado es la que determina a su vez la necesidad de incluir en las competencias, no sólo conocimientos sobre "cómo crear un negocio" o "cómo gestionarlo", sino también habilidades propias del emprendedor (creatividad, espíritu de superación, tolerancia moderada al riesgo, gestión de la incertidumbre, etc.).

Las investigaciones realizadas sobre el impacto de la educación en el emprendimiento no demuestran si la educación estimula o desfavorece la intención de emprender, pues se encuentran evidencias empíricas de ambos casos (Tkachev y Kolvereid, 
1999; Peterman y Kennedy, 2003; Oosterbeek et al, 2010). Lo que sí parece seguro es que la educación permite al estudiante un mejor conocimiento sobre sus posibilidades de emprender y le permite tener una mayor determinación en sus intenciones (von Graevenitz et al, 2010).

Existen estudios que indican que la educación influye en los valores culturales del individuo y, por tanto, en su nivel de emprendimiento (Hayton et al, 2002; Morrison, 2000). Las habilidades transferidas en la educación superior jugarían, por tanto, un importante papel en el establecimiento de características generalmente asociadas al comportamiento emprendedor (Ronstadt, 1985; Vesper, 1982). Sin embargo, la educación orientada exclusivamente a las habilidades no tiene porqué repercutir en la intención de emprender (acción), aunque sí en la actitud hacia el emprendimiento (von Graevenitz et al, 2010).

Parece claro que el foco de la enseñanza debe estar en cómo ser o actuar como emprendedor y no exclusivamente en cuáles son los pasos a seguir para crear una empresa (Gibb, 2002; Gibb y Hannon, 2006). Pero para poder establecer los contenidos más adecuados es necesario conocer antes los resultados de la educación en el emprendimiento (Gorman et al, 1997) a través de su medición observada en el comportamiento, intenciones y habilidades (Kolvereid e Isaksen, 2006).

\section{Objetivo y metodología de la investigación}

El objetivo es, por tanto, medir el impacto de la educación por competencias en emprendimiento sobre la intención de autoempleo o de crear un negocio de los estudiantes universitarios. Un análisis de los estudios realizados sobre el impacto de la formación en el emprendimiento o la intención de emprender revela que existen distintas formas de enfrentarse al problema. Por una parte, podemos clasificar las investigaciones en función del enfoque utilizado para realizar la medida; así, hay autores que se centran en el enfoque de rasgos y utilizan escalas para medir los rasgos característicos del emprendedor sobre una muestra de estudiantes, mientras que otros diseñan escalas que miden los tres factores motivacionales del emprendimiento según la TCP. Por otra parte, se pueden distinguir dos metodologías de estudio comparativo, los que comparan los resultados de dos muestras de estudiantes que han recibido distinta formación y los que realizan únicamente una comparación "ex-ante" y una "ex-post" a la formación.

En nuestro caso se propone un estudio utilizando el enfoque mixto y en dos fases, ex-ante y ex-post al impacto de la educación (von Graevenitz et al, 2010), dentro del marco de la diferencia-en-diferencias propuesto por Oosterbeek et al (2010), es decir, mediante el uso de un grupo de control no expuesto a dicho impacto.

La encuesta se dirige a estudiantes del primer y segundo curso de Administración y Dirección de Empresas (ADE) y, como grupo de control, a alumnos de Derecho de la Universidad Católica San Antonio de Murcia. El número total de encuestas váli- 
das obtenidas fue de 110 alumnos (60 de ADE y 50 de Derecho) a los que se le han tomado los datos para realizar un análisis posterior replicado, terminados sus estudios (fase ex-post o a posteriori).

En una primera fase del estudio se clasifica a los encuestados como emprendedores/no emprendedores y se analizan las diferencias entre los que tienen o no intención de emprender y entre el grupo de ADE y el de Derecho, siendo el siguiente paso el seguimiento de los alumnos encuestados a través de una entrevista que se realizará al finalizar el Grado. Con los resultados obtenidos tras la formación, se comparará el grupo de ADE y el de Derecho y los emprendedores/no emprendedores de la muestra a priori y a posteriori. Se pretende con esto saber el nivel de impacto de la formación en competencias recibida sobre la intención de emprendimiento y en los factores que influyen en ella.

En la muestra se analizan los rasgos que la literatura establece como característicos de los emprendedores: necesidad de logro, autonomía, autosuficiencia percibida, resistencia, propensión moderada al riesgo, tolerancia a la incertidumbre, creatividad e innovación; para, posteriormente y siguiendo la Teoría del Comportamiento Planeado (TCP), evaluar los tres precursores básicos de la intención de emprender: las actitudes personales, las normas subjetivas y el control percibido. Para ello se utilizan escalas ya validadas en anteriores estudios.

Las habilidades y capacidades de los estudiantes para la gestión de proyectos emprendedores se miden a través de la escala usada por Oosterbeek et al. (2010) y para la intención de emprendimiento se utiliza la escala categórica de siete posiciones adaptada de Liñán y Chen (2006). A partir de la escala EIQ (Entrepreneurial Intention Questionnaire), utilizada por Moriano et al (2012), y las aportaciones realizadas por Michail (2000) y Liñán y Chen (2006), se miden las creencias de los sujetos respecto al emprendimiento, la influencia social percibida respecto a esta conducta y las habilidades y capacidades percibidas que condicionan el control del sujeto para llevar a cabo su proyecto emprendedor. En cuanto a la medición de la influencia social respecto a la creación de una empresa propia, se utiliza la escala elaborada por Liñán et al. (2011).

En la última fase (ex-post) se pretende utilizar, además de las anteriores, una encuesta para medir el impacto de la formación percibido por el estudiante basado en la desarrollada por von Graevenitz et al (2010) que incluirá ítems como: "La formación recibida me ha ayudado a comprender mejor las actitudes, valores y motivaciones principales de un emprendedor", "Ha mejorado mi capacidad de gestión y planificación de un futuro negocio", etc. y cuestiones sobre la percepción de barreras al emprendimiento (económicas y sociales) e incentivos. 


\section{Resultados}

En la primera fase del estudio, llevada a cabo en el curso 2012-2013, se han analizado las diferencias entre los que tienen o no intención de emprender y entre el grupo de ADE y el de Derecho. Se ha clasificado como emprendedores a aquellos alumnos que obtienen un índice de intención de emprendimiento superior a 3,5 (mitad de la escala de medida), que en la muestra suponen un $62 \%$ de los alumnos de ADE y un $32 \%$ de los de Derecho.

La observación de las diferencias de respuestas en la escala sobre intención de emprender indica una mayor puntuación en todos los ítems en los alumnos de ADE, siendo la diferencia de medias estadísticamente significativas según el análisis ANOVA a un nivel significativo de $0,05(\mathrm{~F}=7,587$, sig. 0,011$)$. También encontramos una importante diferencia en las creencias sobre las propias capacidades para el emprendimiento. Así, los estudiantes de ADE perciben una mayor autoeficiencia $(3,93)$ que los estudiantes de Derecho $(2,81)$ de forma estadísticamente significativa $(\mathrm{F}=24,90 ;$ sig. 0,001$)$. Aún siendo la muestra de alumnos de primer curso (todavía no han recibido la formación en competencias de emprendimiento), los alumnos de ADE muestran una mejor percepción de sus capacidades y habilidades para crear una empresa; y esto puede ser debido a que muchos de ellos ya han recibido formación en "empresa" (65\%, mientras que en los alumnos de Derecho sólo un 30\%).

Los resultados del análisis realizado confirman el poder de predicción de la Teoría de Comportamiento Planeado (TPC) sobre la intención de emprender en los dos grupos (alumnos de ADE y de Derecho). En la muestra completa, la actitud hacia el emprendimiento, las normas subjetivas y las capacidades y habilidades percibidas son predictores significativos de la intención de emprender (Coeficientes de Correlación Múltiple de Pearson obtenidos entre la puntuación de los tres factores y la de intención de emprendimiento superiores a 0,7 ). Además, los estudiantes de grado en $\mathrm{ADE}$ son más tolerantes al riesgo y asocian el emprendimiento a un mayor nivel de ingresos, siendo estas dos características deseables en un emprendedor $(\mathrm{F}=14,23$; sig. 0,001).

En cuanto a las diferencias entre "emprendedores" de ADE y de Derecho, no se encuentran diferencias significativas entre los dos grupos, salvo en la autoeficiencia percibida, que es mayor en los "emprendedores" de ADE. Las diferencias, además, no son significativas entre emprendedores de ADE y Derecho "con formación en Empresa", pero sí entre los emprendedores "sin formación en Empresa".

\section{Conclusiones}

La comprensión del impacto que causan las distintas competencias integradas en la formación sobre la intención de emprender permite definir con eficacia la metodología para el fomento del emprendimiento desde la formación superior. Por ello, se 
ha diseñado un método de evaluación del impacto en dos fases, anterior y posterior a la formación, y con grupo de control, en el que se tienen en cuenta tanto los rasgos personales que definen al emprendedor como los tres factores desarrollados por la Teoría del Comportamiento Planeado.

Los resultados de la primera fase desarrollada muestran una actitud diferente hacia el emprendimiento, así como una mayor autoeficiencia o control percibido de los alumnos de ADE frente a los de Derecho. Este resultado puede ser explicado por la mayor formación anterior en "empresa" de los alumnos de ADE, que se traduce en una mayor confianza sobre la percepción de sus posibilidades y habilidades de gestión. Por tanto, parece que la formación ha tenido ya un impacto inicial sobre el alumnado, y será interesante conocer la evolución posterior del mismo.

El siguiente paso en la investigación consistirá en la réplica de la encuesta al finalizar el Grado a la misma muestra para su comparación por pares. Se analizarán las diferencias de respuestas entre el grupo de ADE y Derecho, los cambios de respuesta de los clasificados a priori como "emprendedores" y no "emprendedores" y las posibles diferencias de estos grupos según su formación en ADE o Derecho. Se pretende con ello verificar si en el alumno de ADE se produce un cambio en la intención de emprender debido a su formación y el grado o nivel de impacto en comparación con otro tipo de formación en competencias.

\section{Referencias bibliográficas}

AJZEN, I. (2002). "Perceived behavioral control, self-efficacy, locus of control, and the theory of planned behavior", En: Journal of Applied Social Psychology, 32; p. 1-20.

ALDA, R.; VILLARDÓN, L. y ELEXPURU, I. (2012). "Propuesta y validación de un perfil de competencias de la persona emprendedora. Implicaciones para la formación". En: Electronic Journal of Research in Educational Psychology 10 (3); p. 1059-1080.

CANO, C. J.; GARCÍA, J. y GEA, A. B. (2003). Actitudes emprendedoras y creación de empresas en los estudiantes universitarios. Servicio de publicaciones de la Universidad de Almería.

CASSON, M. (1995). Entrepreneurship and Business Culture (Studies in the Economics of Trust)(v. 1). Aldershot (U.K.): Edward Elgar.

COMISIÓN EUROPEA (2004). Implementation of "Education and Training 2010". Work programme. SÄÄVÄLÄ, T.y BALDASSARRI, S. En: Joint subgroup of experts invited by education and culture $D G$ and the enterprise DG. Entrepreneurship education and learning. Directorate General for Education and Culture. November 2004.

(2006). "Entrepreunership education in Europe: foresting entrepreneurial mindsets through education and learning". En: Final proceedings of the Conference 
on Entrepreneurship Education. Oslo: Ministry of Education and Research and Ministry of Trade and Industry.

(2008). "Entrepreneurship in higher education, especially within non-business studies. Final Report of Expert Group" Enterprise and Industry DIRECTORATE-GENERAL. Promotion of SMEs competitiveness Entrepreneurship. March 2008.

GARCÍA, L. et al. (2009). "Diseño de un plan estratégico docente para la formación de estudiantes universitarios hacia la creación y dirección de empresas familiares en Barcelona, España" En V. FERNÁNDEZ (ed.) Nuevas investigaciones sobre la gestión de la empresa familiar en España. OmniaScience; p. 127-144.

GARTNER, W. B. (1985). "A conceptual framework for describing the phenomenon of new venture creation". En: Academy of Management Review, 10 (4); p. 696-706.

GIBB, A. (2002). "Creating conducive environments for learning and entrepreneurship-living with, dealing with, creating and enjoying uncertainty and complexity". En: Industry and Higher Education; p. 135-148.

GIBB, A. y HANNON, P. (2006). "Towards the entrepreneurial university?" En: International Journal of Entrepreneurship Education, 4 (1); p. 73-110.

GLOBAL ENTREPRENEURSHIP MONITOR (2009). Informe ejecutivo GEM España. Informe Anual del Observatorio Global Entrepreneurship Monitor (GEM) sobre Actividad Emprendedora en España. Ministerio de Industria, Comercio y Turismo. Secretaria General de Industria.

GORMAN, G.; HANLON, D. y KING, W. (1997). "Some research perspectives on entrepreneurship education, Enterprise education and education for small business management: a ten year review". En: International Small Business Journal, $15 ;$ p. 56-77.

GÜROL, Y. y ATSAN, N. (2006). "Entrepreneurial characteristics amongst university students: Some insights for entrepreneurship education and training in Turkey". En: Education and Training, 48 (1); p. 25-38.

HAYTON, J. C.; GERARD, G. y SHAKER, A. Z. (2002). "National culture and entrepreneurship: A review of behavioral research". En: Entrepreneurship Theory and Practice 26 (4); p. 33-52.

HOLGREM, C. y FROM, J. (2005). "Taylorism of the mind: Entrepreneurship education from a perspective of educational research". En: European Educational Research Journal, 4 (4); p. 382-390.

KIRZNER, I. M. (1979). Perception, Opportunity and Profit. Chicago: University of Chicago Press.

KOLVEREID, L. y ISAKSEN, E. (2006). "New business start-up and subsequent entry into self-employment".En: Journal of Business Venturing, 21; p. 866-885.

KYRÖ, P. y CARRIER, C. (2005). Entrepreneurial learning in universities: Bridges across borders. En Kyrö, P. y C. Carrier (eds.): The dynamics of learning entrepreneurship in a cross-cultural university context. Hämmeenlinna: University of Tampere; p. 14-43. 
LEE, S. H. y WONG, P. K. (2004). “An exploratory study of technopreneurial intentions: A career anchor perspective".En: Journal of Business Venturing, 19 (1); p. 7-28.

LIÑÁN, F. y CHEN, Y. (2006). Testing the entrepreneurial intention model on a two-country simple. Document de Treball $\mathrm{n}^{\circ}$ 06/7. Universitat Autònoma de Barcelona.

LIÑÁN, F.; URBANO, D. y GUERRERO, M. (2011). "Regional variations in entrepreneurial cognitions: Start-up intentions of university students in Spain". En: Entrepreneurship \& Regional Development, 23(3-4), p. 187-215.

MICHAIL, K. (2000). "Exploring perceptions of a priori barriers to entrepreneurship: a multidisciplinary approach". En: Entrepreneurship: Theory \& Practice, Winter; p. 59-79.

MORIANO, J. A. et al. (2012). "A cross-cultural approach to understanding entrepreneurial intention". Journal of Career Development, 39(2); p. 162-185.

MORRISON, A. (2000). "Entrepreneurship: what triggers it?" En: International Journal of Entrepreneurial Behavior Research, 6(2); pp. 59-71.

OCDE (2008): Entrepreneurship and Higher education. Local Economic and Employment Development Programme (LEED). Paris.

OOSTERBEEK, H.; Van PRAAG, M. y IJSSELSTEIN, A. (2010). "The impact of entrepreneurship education on entrepreneurship skills and innovation" En: European Economic Review 54; p. 442-454.

PARKER, S. C. (2005). "Explaining regional variations in entrepreneurship as multiple occupational equilibria". En: Journal of Regional Science, 45; p. 829-850.

PETERMAN, N. y KENNEDY, J. (2003). "Enterprise education: influencing students' perceptions of entrepreneurship". En: Entrepreneurship Theory and Practice 28; p. 129-144.

RONSTADT, R. (1985). "The educated entrepreneurs: A new era of entrepreneurial education is beginning". En: American Journal of Small Business, summer; p. 7-23.

THOMAS, A. S. y MUELLER, S. L. (2000). "A case for comparative entrepreneurship: Assessing the relevance of culture". En: Journal of International Business Studies, 31; p. 287-301.

TKACHEV, A. y KOLVEREID, L. (1999). "Self-employment intentions among Russian students". En: Entrepreneurship and Regional Development, 11; p. 269-280.

ULIJN, J.M.; DRILLON, D. y LASCH, F. (2007). "Entrepreneurship and cooperation: the emergence and survival of high tech ventures in Europe". En ULIJN, DRLLON y LASCH (eds.), Entrepreneurship, Cooperation and the Firm: The Emergence and Survival of High Tech Ventures in Europe, Cheltenham: Edward Elgar.

Van PRAAG, M. C. (1999). "Some classic views on entrepreneurship". En: Economist 147 (3); p. 311-335.

(2007): "What is the value of entrepreneurship? A review of recent research". En: Small Business Economics 29 (4); p. 351-382. 
VECIANA, J. M. (1999). "Creación de Empresas como programas de Investigación Científica". En: Revista de Económica Europea de Dirección y Creación de Empresas, 8(3); p. 11-36.

VESPER, K. H. (1982): "Introduction and summary of entrepreneurship research". En C.A. KENT, D.L.; SEXTON, K.H. y ENGLEWOOD, C. (eds.): Encyclopedia of entrepreneurship. Prentice Hall; p. 425.

Von GRAEVENITZ, G.; HARHOFF, D. y WEBER, R. (2010). "The effects of entrepreneurship education". En: Journal of Economic Behavior and Organization 76 (1); p. 90-112.

WANDOSELL; G. y PALAO, J. (2012). "El perfil del director/directivo del futuro" En: Revista Derecho y Empresa. Aranzadi, 1; p. 185-199.

WENNEKERS, S. et al. (2005). "Nascent entrepreneurship and the level of economic development". En: Small Business Economics, 24; p. 293-309.

YÁNIZ, C. y VILLARDÓN, L. (2006). Cuadernos Monográficos del ICE: Planificar desde competencias para promover el aprendizaje. El reto de la sociedad del conocimiento para el profesorado universitario. Volumen 12: Bilbao: Publicaciones de la Universidad de Deusto.

\section{Los autores}

Flores Asenjo, M. Pilar. Doctora en Economía y Medio Ambiente en 2008 por la Universidad Politécnica de Valencia. Profesora del Departamento de Administración y Dirección de Empresas de la Universidad Católica San Antonio de Murcia, miembro del Grupo de Investigación "Organización de empresas y marketing", coordinadora departamental de Relaciones Internacionales de la UCAM y miembro del cuerpo de tutores.

Palao Barberá, José. Ingeniero Superior de Telecomunicaciones en 1982 por la Universidad Politécnica de Madrid. Máster en MBA en 2013 por la UCAM. Director del Máster Universitario en Dirección y Gestión de Sistemas de Calidad y Medio Ambiente. Profesor del Departamento de Administración y Dirección de Empresas de la Universidad Católica San Antonio de Murcia. 